\title{
Critical Landau Velocity in Helium Nanodroplets
}

\author{
Nils B. Brauer, ${ }^{1}$ Szymon Smolarek, ${ }^{2, *}$ Evgeniy Loginov, ${ }^{1, \dagger}$ David Mateo, ${ }^{3}$ Alberto Hernando, ${ }^{3, *}$ Marti Pi, ${ }^{3}$ \\ Manuel Barranco, ${ }^{3}$ Wybren J. Buma, ${ }^{2}$ and Marcel Drabbels ${ }^{1, \S}$ \\ ${ }^{1}$ Laboratoire de Chimie Physique Moléculaire, Ecole polytechnique Fédérale de Lausanne (EPFL), CH-1015 Lausanne, Switzerland \\ ${ }^{2}$ Faculty of Science, Van't Hoff Institute for Molecular Sciences, University of Amsterdam, Science Park 904, \\ 1098 XH Amsterdam, Netherlands \\ ${ }^{3}$ Departament ECM, Facultat de Física, and $\mathrm{IN}^{2} \mathrm{UB}$, Universitat de Barcelona, 08028 Barcelona, Spain
}

(Received 6 May 2013; published 8 October 2013)

\begin{abstract}
The best-known property of superfluid helium is the vanishing viscosity that objects experience while moving through the liquid with speeds below the so-called critical Landau velocity. This critical velocity is generally considered a macroscopic property as it is related to the collective excitations of the helium atoms in the liquid. In the present work we determine to what extent this concept can still be applied to nanometer-scale, finite size helium systems. To this end, atoms and molecules embedded in helium nanodroplets of various sizes are accelerated out of the droplets by means of optical excitation, and the speed distributions of the ejected particles are determined. The measurements reveal the existence of a critical velocity in these systems, even for nanodroplets consisting of only a thousand helium atoms. Accompanying theoretical simulations based on a time-dependent density functional description of the helium confirm and further elucidate this experimental finding.
\end{abstract}

DOI: 10.1103/PhysRevLett.111.153002

PACS numbers: 33.80.- b, 36.40.- c, 67.25.dw

Analogous to superconductivity, superfluidity is a macroscopic manifestation of quantum mechanics. It derives its name from the frictionless flow of a liquid [1,2]. While superfluidity has been observed for Bose-Einstein condensates [3] and more recently for polaritons, [4] helium is undoubtedly the best-known example of a superfluid. The peculiar dispersion curve of $\mathrm{He}$ dictates that an object moving through superfluid helium can only create elementary excitations if its speed exceeds the so-called critical Landau velocity of $\sim 58 \mathrm{~m} / \mathrm{s}[5,6]$. Whereas the critical Landau velocity could be experimentally verified in bulk helium, [7] its manifestation in finite size helium systems is still matter of debate [8-10]. Knowledge of such a fundamental property becomes essential as finite size helium systems, in the form of helium nanodroplets, are increasingly being used as a matrix for a wide variety of studies [11-13].

Many properties of helium nanodroplets have been characterized during the last two decades using solvated molecules as spectroscopic probes [14]. Vibrational spectroscopy of solvated carbonyl sulfide (OCS) has provided evidence for microscopic superfluidity in these finite size systems [15]. While a clearly resolved rotational structure was observed in the IR absorption spectrum of OCS in ${ }^{4} \mathrm{He}$ droplets, this structure was markedly absent in ${ }^{3} \mathrm{He}$ droplets, which are not superfluid due to their fermionic character. In contrast, the temporal evolution of rotational wave packets of methyliodide molecules dissolved in helium droplets has recently been found to differ dramatically from that of isolated molecules [16]. This raises the question to what extent microscopic superfluidity can be related to the frictionless flow of superfluid helium. Here, we present an approach that uses the translational motion of electronically excited atoms and molecules to probe superfluidity in helium nanodroplets and to establish the existence of a critical velocity in these finite size systems.

Most atoms and molecules in their electronic ground state experience a weakly attractive interaction with helium. As a result, these species are located in the interior of helium droplets [17]. Since electrons experience a repulsive short-range interaction with helium, the interaction of an atom or molecule with helium can become repulsive upon electronic excitation. In such a case, an excited impurity will be accelerated towards the surface and might eventually be expelled from the droplet. Rydberg states of atoms and molecules, having extended electron orbits, are prototypical examples of states having such repulsive interactions [18-22]. Because of the relatively small repulsive interaction energies associated with the excitations of the atoms and molecules, the velocity distributions of the ejected impurities might carry a signature of the critical velocity in these finite size systems. This was not possible in previous experiments using photofragments as probes because of the large energy released by the dissociation process [23]. In the present investigation a wide variety of probes have been used, ranging from atomic $\mathrm{Ag}$, to diatomic NO, to nearly planar trimethylamine (TMA), to the three-dimensional cage amines 1,4-diazabicyclo[2.2.2] octane (DABCO) and 1-azabicyclo[2.2.2]octane (ABCO), as to ascertain that the characteristics of the velocity distribution are universal and not specific to one type of system.

The experiments have been performed using a setup that has been described in detail before [24,25]. A schematic 


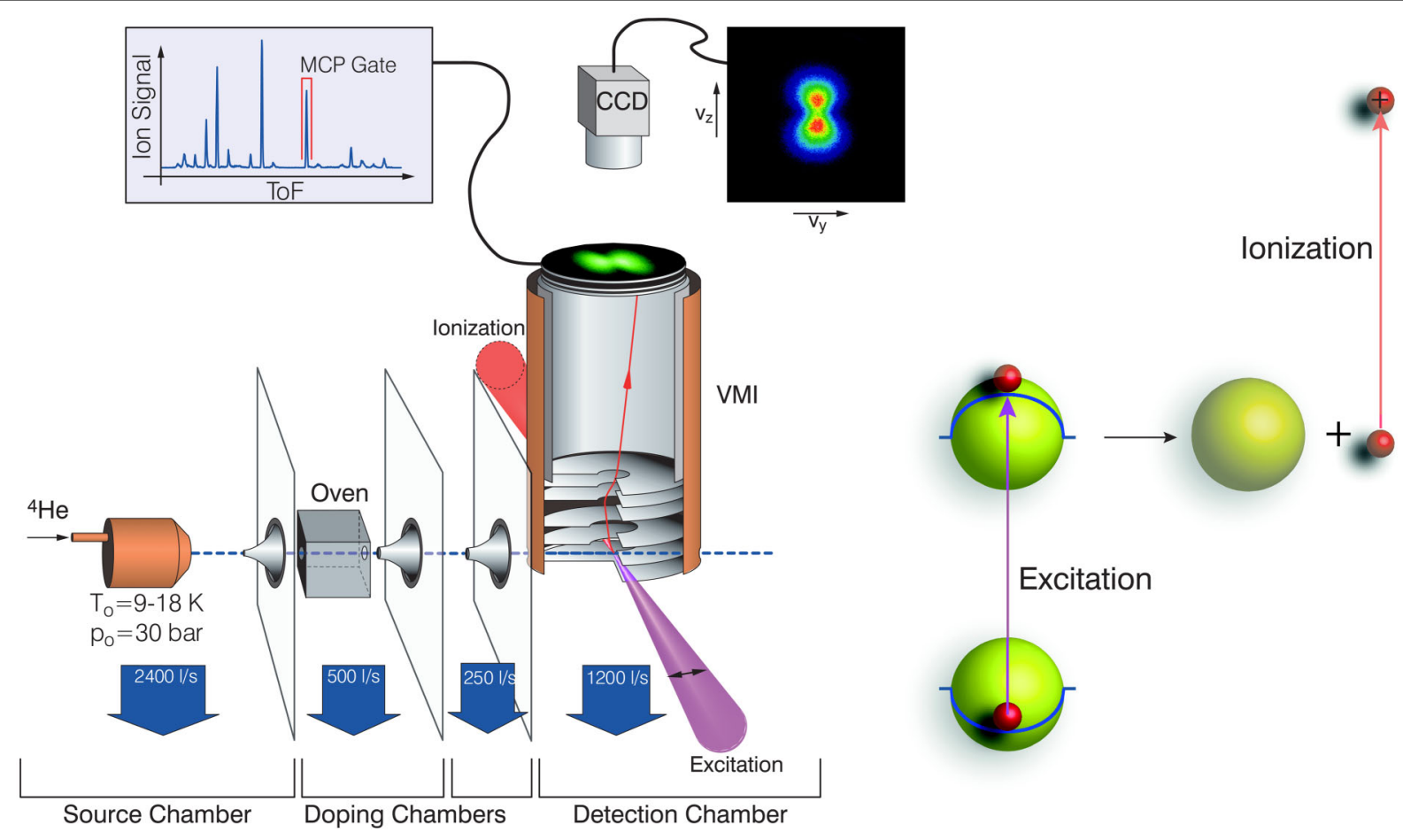

FIG. 1 (color online). Overview of the experimental setup (left) and diagram representing the experimental method (right). Following excitation of a solvated impurity to a state having a repulsive interaction with the helium, the excited species is ejected from the droplets and subsequently ionized by the absorption of an additional photon.

overview of the setup and method used is presented in Fig. 1. Helium nanodroplets are formed by expanding helium gas at 30 bar into vacuum through a $5 \mu \mathrm{m}$ aperture cooled to temperatures in the range of $9-20 \mathrm{~K}$. The droplets consist on average of $1 \times 10^{3}-5 \times 10^{6}$ helium atoms, depending on the temperature of the nozzle $[26,27]$. The helium droplets pick up atoms or molecules as they pass through a vapor of the species of interest. Via a differential pumping stage the doped droplets enter a velocity map imaging (VMI) spectrometer [28]. At the center of the spectrometer, the droplet beam is crossed perpendicularly by the linearly polarized output of one or two Nd:YAG pumped dye lasers. The embedded species are electronically excited by the absorption of a resonant photon. After their ejection from the droplets the excited species are ionized by the absorption of an additional photon, either from the same or from another laser. The resulting ions are projected onto a position-sensitive detector. By performing an inverse Abel transformation on the recorded images the three-dimensional velocity distribution of the ions is determined [29].

The experiments are complemented by simulations of the translational dynamics of excited $\mathrm{Ag}$ atoms in helium droplets consisting of 1000 atoms. Details of these calculations are published elsewhere [30]. Briefly, the calculations are based on a three-dimensional, time-dependent density functional approach for the helium, while the silver atom is treated classically. To keep the calculations computationally affordable the Orsay-Trento (OT) density functional for the helium has been used neglecting the backflow term [31]. This term has no effect on the static properties of the system but affects its dynamics in such a way that the Landau velocity is overestimated to $v_{L}=$ $96 \mathrm{~m} / \mathrm{s}$ instead of the $v_{L}=58 \mathrm{~m} / \mathrm{s}$ measured for bulk helium [31].

We will discuss the experimental results obtained for silver and nitric oxide in detail here. The other systems investigated show similar characteristics and where relevant these will be mentioned. The $\mathrm{Ag}-\mathrm{He}_{N}$ system has been investigated in detail $[22,25]$. It has been found that following excitation of the silver atom via the $5 p^{2} P_{1 / 2} \leftarrow$ $5 s^{2} S$ transition nearly all excited atoms are ejected from the droplets. In contrast, after excitation of the other spinorbit state via the $5 p^{2} P_{3 / 2} \leftarrow 5 s^{2} S$ transition, a significant fraction of the atoms remains solvated, while most of those that are ejected have relaxed to the $5 p^{2} P_{1 / 2}$ state. The $\mathrm{NO}-\mathrm{He}_{N}$ system is less well characterized. In the present study the embedded NO molecule is excited to the $A^{2} \Sigma^{+}$ state by the absorption of a single UV photon. In agreement with previous work, the one-photon excitation spectrum reveals a large blue-shift of several thousand wave numbers [32]. The ionization spectrum of the excited NO $A^{2} \Sigma^{+}$ provides conclusive evidence that the NO molecules leave the helium droplets in the electronically excited $A^{2} \Sigma^{+}$state since its onset coincides with that of free $\mathrm{NO}$, this in contrast to spectra of solvated species $[33,34]$. The other molecules used in this study, TMA, DABCO, and ABCO, have not 


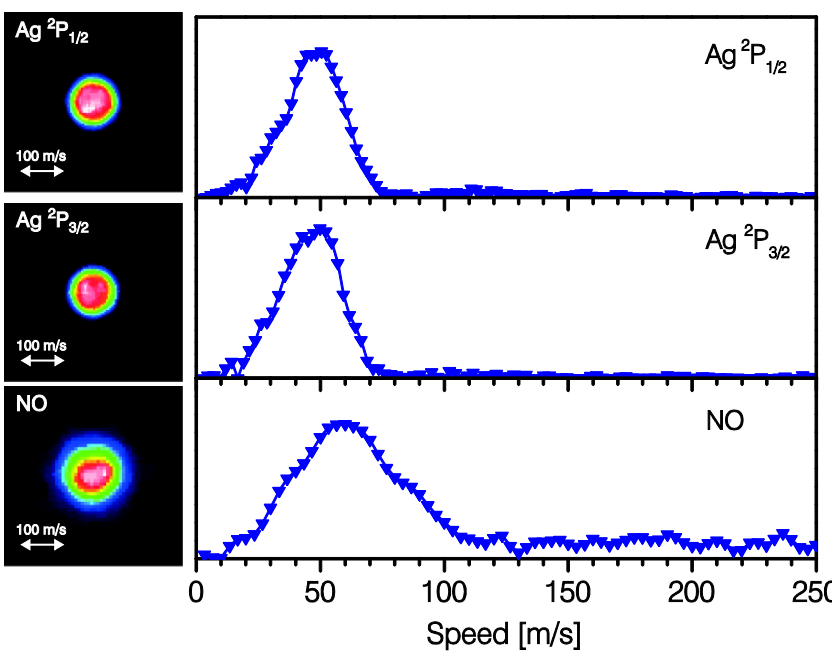

FIG. 2 (color online). Velocity map images and corresponding speed distributions of $\mathrm{Ag}$ and $\mathrm{NO}$ ejected from helium droplets with a mean radius of $22.2 \mathrm{~nm}$ following their excitation to the ${ }^{2} P_{1 / 2},{ }^{2} P_{3 / 2}$, and $A^{2} \Sigma^{+}$state, respectively.

been investigated before by helium droplet spectroscopy. The lowest excited singlet states of these molecules are of Rydberg character with the $2 p$ electron of the nitrogen atom excited to the $3 s$ or the $3 p$ orbital [35-38]. Excitation to these states results in the ejection of the molecules from the helium droplets, as has been explicitly checked for by photoelectron spectroscopy.

Prototypical ion images of $\mathrm{Ag}$ and $\mathrm{NO}$ recorded after expulsion from helium droplets consisting of on average of $1 \times 10^{6}$ atoms, corresponding to a mean droplet radius of $22.2 \mathrm{~nm}$, are presented in Fig. 2. The images reveal that the excited species leave the droplets with isotropic angular distributions and with very similar speeds. This is confirmed by the speed distributions that have been extracted from these images and that are also shown in Fig. 2. It should be noted here that the speed distribution for $\mathrm{NO}$ is blurred beyond the resolution of the setup, $10 \mathrm{~m} / \mathrm{s}$, due to the ion recoil in the ionization process. We also find that the distributions depend only very weakly on the size of the droplets. In order to highlight the similarity of the velocity distributions of the different species, the most probable speeds are plotted in Fig. 3 as a function of droplet size. For all species the most probable speed lies in the range of 40-60 m/s and increases with increasing droplet size up to a helium droplet radius of $6 \mathrm{~nm}$ after which it stays nearly constant. These results indicate that the motion of the excited species through the helium is largely independent of its mass and of the distance traveled through the helium.

In view of the quite different interaction strengths of the excited atoms and molecules with the helium, as well as the differences in masses and geometries, rather different speed distributions for the ejected species are expected. This is best exemplified by comparing Ag and NO. The excited state solvation energies for these species have been

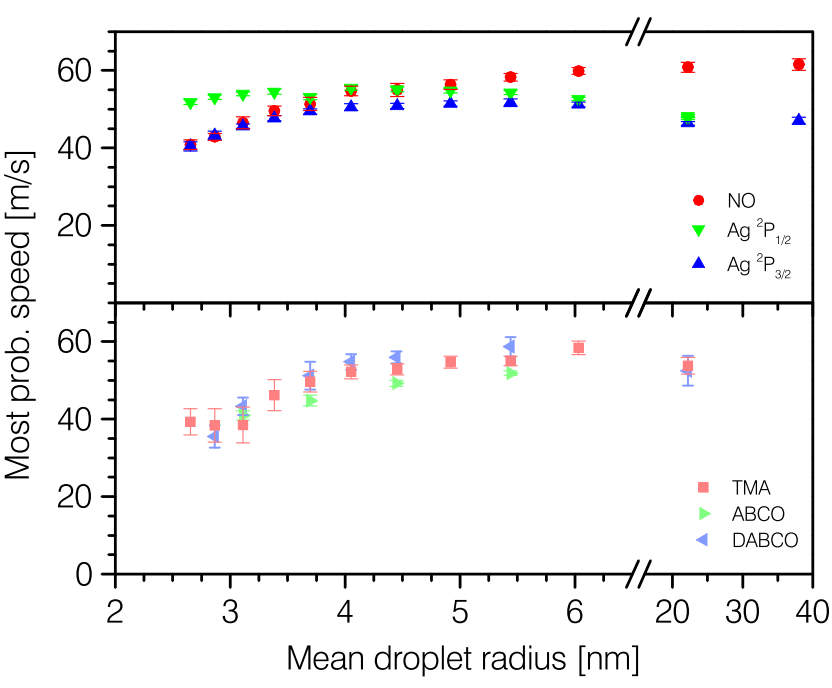

FIG. 3 (color online). The most probable speeds of the various dopants after their ejection from helium droplets of different sizes.

calculated to be 62 and $93 \mathrm{~cm}^{-1}$, respectively $[39,40]$. In the absence of dissipation, this energy is fully converted into kinetic energy, yielding final speeds of 120 and $270 \mathrm{~m} / \mathrm{s}$ for $\mathrm{Ag}$ and NO, respectively. As these speeds are substantially larger than those observed experimentally, it has to be concluded that the speeds are limited by dissipation. It is unlikely that viscous drag is the origin of the dissipation as this would require a much larger drag for $\mathrm{NO}$ than for $\mathrm{Ag}$ while their solvation structures are very similar. Consequently, the remarkable fact that the observed speed distributions are all very similar indicates that there exists a critical velocity for the motion of the excited species in helium droplets.

The weak droplet size dependence observed for the speed distributions is in line with this assumption. Following excitation of the dopants the excess energy $E_{\text {exc }}$, defined as the difference between the photon energy and the sum of the solvation energy of the unexcited impurity and the energy of the ejected impurity in free space, is transferred to the helium. The $\mathrm{AgHe}_{1000}$ simulations reveal that this energy transfer occurs on a sub-picosecond time scale. This prompt energy input leads to a heating of the droplets, but in most cases their temperature remains below the superfluid transition temperature. Although these small droplets are expected to remain superfluid, the superfluid fraction will be reduced [41]. Consequently, the excited species will experience drag as they are being accelerated out of the droplets and their velocity distributions are shifted towards low speeds. This effect is most notable for those systems having a large excess energy, like $\mathrm{NO}\left(E_{\mathrm{exc}}=3600 \mathrm{~cm}^{-1}\right)$ and DABCO $\left(E_{\mathrm{exc}}=\right.$ $\left.4300 \mathrm{~cm}^{-1}\right)$. The fact that the speed distributions for large droplets become droplet size independent for all systems investigated here indicates that in these droplets the nonsuperfluid fraction is negligible. We therefore will focus our discussion on the speed distributions of impurities expelled from the largest droplets used in this study. The characteristic 


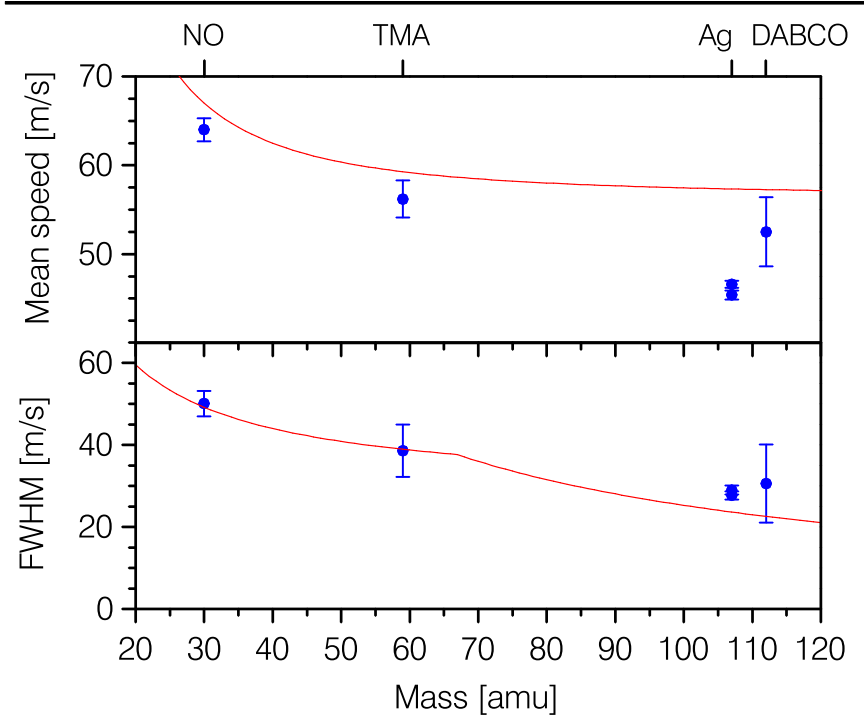

FIG. 4 (color online). Experimental (circles) and theoretical (lines) mean speed and width (FWHM) of the speed distributions as a function of dopant mass using helium droplets with a mean radius of $22.2 \mathrm{~nm}$.

properties of these speed distributions, i.e., mean speed and width, are presented in Fig. 4. The data in this figure indicate that both properties decrease with the mass of the dopant.

Drift experiments on negative ions in bulk helium have identified two different dissipation processes, i.e., vortex nucleation and the creation of roton pairs $[7,42,43]$. The critical velocities associated with these processes are quite similar. In contrast, their rates differ by orders of magnitude. While the vortex nucleation rates are below $10^{6} \mathrm{~s}^{-1}$, the rates for roton pair creation readily exceed $10^{10} \mathrm{~s}^{-1}$. Assuming that the properties of finite size and bulk helium are similar, [41] this large difference can be used to discriminate between the two processes in the present experiment. Time-resolved pump-probe experiments reveal that excited $\mathrm{Ag}$ atoms leave the droplets on a sub-nano-second time scale, even for the largest droplets considered in this study. Simulations on the $\mathrm{AgHe}_{1000}$ system are in line with this observation, as they indicate that the excited Ag impurity desorbs from the helium droplet on a sub-100 ps time scale. Based on these results, vortex nucleation is ruled out as the origin of the critical velocity in helium droplets of the sizes used in this study. We therefore propose that the existence of the critical velocity is the result of roton pair creation by the moving impurity. Since the roton pair creation process is fully determined by the dispersion curve of superfluid helium, a direct comparison between experiment and theory becomes possible.

The velocity of an object in superfluid helium under the influence of a force will increase with time until it reaches the critical Landau velocity, $v_{\max }$, at which point a pair of rotons is created and the velocity reduces to $v_{\min }$ [7]. This process will repeat itself, in our case until the object has left the droplet. Assuming that roton pair creation occurs instantaneously when the critical velocity is reached, conservation of energy and momentum dictates that the velocity is limited to the range defined by

$$
\mathrm{v}_{\max / \min }=\frac{\varepsilon}{\hbar k} \pm \frac{\hbar k}{m},
$$

where $m$ is the mass of the object and $v_{\max }$ and $v_{\min }$ are the speeds just before and after the creation of two rotons, each with energy $\varepsilon$ and momentum $k$ [7]. Equation (1) has been evaluated using parameters corresponding to a temperature of $0.5 \mathrm{~K}$ and pressure of $2 \mathrm{bar}$, [6] as these conditions match most closely the properties of the helium droplets [14]. Taking into account that the flux of ejected impurities is speed dependent, one finds that the theoretical average speed of the detected species is given by

$$
\overline{\mathrm{v}}=\frac{\varepsilon}{\hbar k}+\frac{1}{3}\left(\frac{\hbar k}{m}\right)^{2} \frac{\hbar k}{\varepsilon}
$$

and the full width at half maximum of the speed distributions by

$$
\Delta \mathrm{v}=\min \left[\frac{1}{2}\left(\frac{\varepsilon}{\hbar k}+\frac{\hbar k}{m}\right), 2\left(\frac{\hbar k}{m}\right)\right] .
$$

Inspection of Fig. 4 reveals that while the calculated widths of the distributions are in quantitative agreement with experiment, the calculated mean speeds are slightly larger than experimentally observed.

The time-dependent density functional calculations on $\mathrm{AgHe}_{1000}$ provide insight into this difference. The phase space trajectory of excited $\mathrm{Ag}^{2} P_{1 / 2}$ shown in Fig. 5 reveals that following the initial acceleration of the $\mathrm{Ag}$ atom its speed slightly decreases in the surface region of the droplet. While the interaction of the excited Ag atom with the helium droplet is repulsive inside the droplet, it becomes weakly attractive at the surface region; see Fig. 5. The maximum speed is not reached when the silver atom is at the position corresponding to the minimum of the potential well, but while it is still in the interior of the droplet. This

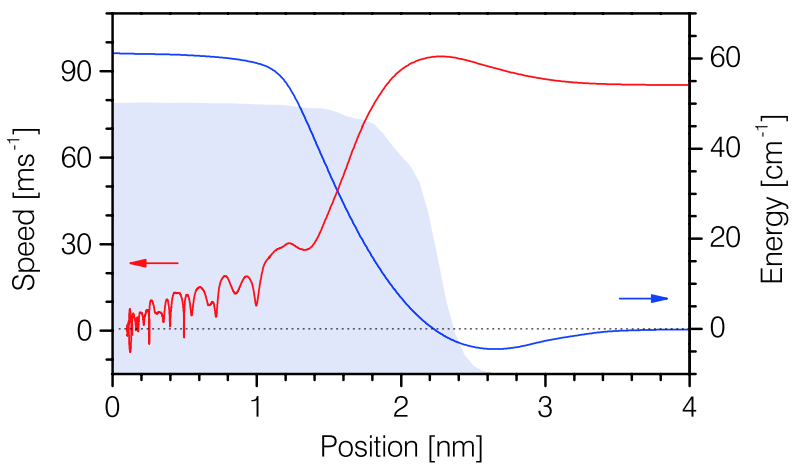

FIG. 5 (color online). Energy of ${ }^{2} P_{1 / 2}$ excited $\mathrm{Ag}$ as a function of position with respect to the center of a $\mathrm{He}_{1000}$ droplet and calculated speed of $\mathrm{Ag}$ following excitation to the ${ }^{2} P_{1 / 2}$ state. The shaded area indicates the helium density profile of a pure $\mathrm{He}_{1000}$ droplet. 
suggests that either dissipative forces are still acting on the $\mathrm{Ag}$ in the low density surface region, or that the system does not follow the potential curve adiabatically. If one assumes the former, the average speed of the Ag atom inside the droplet before desorption can be determined from the experimental data by adding the potential well energy of $4.5 \mathrm{~cm}^{-1}$ to the measured kinetic energy. The resulting speed of $56 \mathrm{~m} / \mathrm{s}$ matches the theoretical result nearly exactly, suggesting that the small difference between the theoretical and experimental results is related to the presence of a small potential well in the surface region. The fact that the maximum speed obtained in the simulations is higher than experimentally observed, see Fig. 5, can be attributed to the particular helium density functional used in the calculations which yields a critical velocity of $v_{L}=96 \mathrm{~m} / \mathrm{s}$, see above [31].

Having shown that the speed distributions of impurities ejected from droplets consisting of $\sim 10^{6}$ helium atoms are in agreement with the existence of a critical Landau velocity, the question arises as to how many atoms are actually required for this concept to be valid. It should be kept in mind that heating of the helium droplets by excitation of the dopant will modify the speed distributions of the dopants. Contrary to the other systems investigated here, excitation of $\mathrm{Ag}$ to the ${ }^{2} P_{1 / 2}$ state releases very little energy, $\sim 440 \mathrm{~cm}^{-1}$, into the droplets and consequently $\mathrm{Ag}$ atoms can be used to address this question [25]. Figure 3 reveals that the speed distribution of $\operatorname{Ag}^{2} P_{1 / 2}$, in contrast to the other species, varies very little with droplet size. We take this as evidence that a critical velocity exists for all droplet sizes used in this study down to those consisting on average of $\sim 1000$ atoms [44]. Although, in a strict sense the present experiments do not prove the existence of a critical Landau velocity-for this similar experiments involving ${ }^{3} \mathrm{He}$ droplets would be required - the observation of a limiting velocity and the good agreement between the observed speed distributions and those of model calculations based on the creation of roton pairs by the moving impurity strongly suggest that a critical Landau velocity does exist in helium droplets, even for those containing as few as 1000 helium atoms.

We gratefully acknowledge the financial support provided by the Swiss National Science Foundation (Grant No. 200020_129623), The Netherlands Organization for Scientific Research (NWO), the DGI, Spain (Grant No. FIS2011-28617-C02-02) and the Generalitat de Catalunya (Grant No. 2009SGR1289).

*Present address: ASML B.V., 5504 DR Veldhoven, Netherlands.

'Present address: SICPA SA, 1000 Lausanne, Switzerland. Present address: Laboratory of Theoretical Physical Chemistry, Ecole polytechnique Fédérale de Lausanne (EPFL), CH-1015 Lausanne, Switzerland.
${ }^{\S}$ Corresponding author.

Marcel.Drabbels@epfl.ch

[1] P. Kapitza, Nature (London) 141, 74 (1938).

[2] J.F. Allen and A. D. Misener, Nature (London) 141, 75 (1938).

[3] C. Raman, M. Kohl, R. Onofrio, D. S. Durfee, C. E. Kuklewicz, Z. Hadzibabic, and W. Ketterle, Phys. Rev. Lett. 83, 2502 (1999).

[4] A. Amo, D. Sanvitto, F. P. Laussy, D. Ballarini, E. del Valle, M. D. Martin, A. Lemaitre, J. Bloch, D. N. Krizhanovskii, M.S. Skolnick, C. Tejedor, and L. Vina, Nature (London) 457, 291 (2009).

[5] L. Landau, Phys. Rev. 60, 356 (1941).

[6] M. R. Gibbs, K.H. Andersen, W. G. Stirling, and H. Schober, J. Phys. Condens. Matter 11, 603 (1999).

[7] D. R. Allum, P. V.E. Mcclintock, A. Phillips, and R. M. Bowley, Phil. Trans. R. Soc. A 284, 179 (1977).

[8] J. Harms and J.P. Toennies, Phys. Rev. Lett. 83, 344 (1999).

[9] M. Fárník, U. Henne, B. Samelin, and J. P. Toennies, Phys. Rev. Lett. 81, 3892 (1998).

[10] M. Schlesinger, M. Mudrich, F. Stienkemeier, and W. T. Strunz, Chem. Phys. Lett. 490, 245 (2010).

[11] A. Gutberlet, G. Schwaab, O. Birer, M. Masia, A. Kaczmarek, H. Forbert, M. Havenith, and D. Marx, Science 324, 1545 (2009).

[12] F. Bierau, P. Kupser, G. Meijer, and G. von Helden, Phys. Rev. Lett. 105, 133402 (2010).

[13] E. Loginov, L.F. Gomez, N. Chiang, A. Halder, N. Guggemos, V. V. Kresin, and A.F. Vilesov, Phys. Rev. Lett. 106, 233401 (2011).

[14] J. P. Toennies and A. F. Vilesov, Angew. Chem., Int. Ed. 43, 2622 (2004).

[15] S. Grebenev, J. P. Toennies, and A. F. Vilesov, Science 279, 2083 (1998).

[16] D. Pentlehner, J. H. Nielsen, A. Slenczka, K. Molmer, and H. Stapelfeldt, Phys. Rev. Lett. 110, 093002 (2013).

[17] F. Ancilotto, P. B. Lerner, and M. W. Cole, J. Low Temp. Phys. 101, 1123 (1995).

[18] E. Loginov, C. Callegari, F. Ancilotto, and M. Drabbels, J. Phys. Chem. A 115, 6779 (2011).

[19] L. Fechner, B. Gruner, A. Sieg, C. Callegari, F. Ancilotto, F. Stienkemeier, and M. Mudrich, Phys. Chem. Chem. Phys. 14, 3843 (2012).

[20] A. Hernando, M. Barranco, M. Pi, E. Loginov, M. Langlet, and M. Drabbels, Phys. Chem. Chem. Phys. 14, 3996 (2012).

[21] A. Kautsch, M. Hasewend, M. Koch, and W.E. Ernst, Phys. Rev. A: At., Mol., Opt. Phys. 86, 033428 (2012).

[22] F. Federmann, K. Hoffmann, N. Quaas, and J. D. Close, Phys. Rev. Lett. 83, 2548 (1999).

[23] A. Braun and M. Drabbels, Phys. Rev. Lett. 93, 253401 (2004).

[24] A. Braun and M. Drabbels, J. Chem. Phys. 127, 114303 (2007).

[25] E. Loginov and M. Drabbels, J. Phys. Chem. A 111, 7504 (2007).

[26] M. Lewerenz, B. Schilling, and J.P. Toennies, Chem. Phys. Lett. 206, 381 (1993).

[27] L. F. Gomez, E. Loginov, R. Sliter, and A. F. Vilesov, J. Chem. Phys. 135, 154201 (2011). 
[28] A. T. J. B. Eppink and D. H. Parker, Rev. Sci. Instrum. 68, 3477 (1997).

[29] M. J. J. Vrakking, Rev. Sci. Instrum. 72, 4084 (2001).

[30] D. Mateo, A. Hernando, M. Barranco, E. Loginov, M. Drabbels, and M. Pi, Phys. Chem. Chem. Phys., DOI: $10.1039 / C 3 C P 52221 \mathrm{~K}$ (to be published).

[31] F. Dalfovo, A. Lastri, L. Pricaupenko, S. Stringari, and J. Treiner, Phys. Rev. B 52, 1193 (1995).

[32] E. Polyakova, D. Stolyarov, and C. Wittig, J. Chem. Phys. 124, 214308 (2006).

[33] E. Loginov and M. Drabbels, Phys. Rev. Lett. 106, 083401 (2011).

[34] E. Loginov, D. Rossi, and M. Drabbels, Phys. Rev. Lett. 95, 163401 (2005).

[35] S. T. Pratt, Chem. Phys. Lett. 360, 406 (2002).

[36] D. H. Parker and P. Avouris, Chem. Phys. Lett. 53, 515 (1978).

[37] E. Tannenbaum, E. M. Coffin, and A. J. Harrison, J. Chem. Phys. 21, 311 (1953).
[38] H. Sato, M. Kawasaki, K. Toya, K. Sato, and K. Kimura, J. Phys. Chem. 91, 751 (1987).

[39] F. Cargnoni and M. Mella, J. Phys. Chem. A 115, 7141 (2011).

[40] J. Klos, G. Chalasinski, M. T. Berry, R. Bukowski, and S. M. Cybulski, J. Chem. Phys. 112, 2195 (2000).

[41] P. Sindzingre, M. L. Klein, and D. M. Ceperley, Phys. Rev. Lett. 63, 1601 (1989).

[42] R. M. Bowley, P. V.E. Mcclintock, F.E. Moss, G. G. Nancolas, and P.C.E. Stamp, Phil. Trans. R. Soc. A 307, 201 (1982).

[43] C. M. Muirhead, W.F. Vinen, and R. J. Donnelly, Phil. Trans. R. Soc. A 311, 433 (1984).

[44] This number is based on the expected droplet size from the helium stagnation conditions and by taking into account the evaporation of helium atoms due to the collision and solvation energy released into the helium droplets upon pickup of the Ag atom. 